\title{
THE ST. LOUIS MEETING OF THE AMERICAN MATHEMATICAL SOCIETY.
}

THE thirteenth western meeting of the American Mathematical Society, combining the forty-fourth regular meeting of the Chicago Section, and the twelfth regular meeting of the Southwestern Section, was held at the Soldan High School, St. Louis, Missouri, on Tuesday and Wednesday, December 30 and 31. On Tuesday afternoon there was a joint session with Section A of the American Association for the Advancement of Science, and the Missouri Section of the Mathematical Association of America.

The attendance at this meeting included fifty persons, among whom were the following forty-four members of the society:

Professor N. Altshiller-Court, Professor C. H. Ashton, Professor G. D. Birkhoff, Professor G. A. Bliss, Professor H. Blumberg, Professor W. C. Brenke, Dr. C. C. Camp, Professor A. B. Coble, Professor S. C. Davisson, Professor E. L. Dodd, Professor A. Dresden, Professor O. Dunkel, Professor H. J. Ettlinger, Professor Tomlinson Fort, Dr. J. B. Glasgow, Professor H. Halperin, Professor E. R. Hedrick, Professor T. H. Hildebrandt, Professor G. O. James, Professor H. E. Jordan, Professor O. D. Kellogg, Professor Solomon Lefschetz, Professor A. C. Lunn, Dr. G. F. McEwen, Professor J. V. McKelvey, Professor T. E. Mason, Professor G. A. Miller, Professor B. E. Mitchell, Professor U. G. Mitchell, Professor F. R. Moulton, Dr. J. R. Musselman, Dr. C. A. Nelson, Professor B. L. Newkirk, Professor P. R. Rider, Professor H. L. Rietz, Professor W. J. Risley, Professor W. H. Roever, Mr. J. B. Rosenbach, Dr. G. W. Smith, Professor R. B. Stone, Professor E. B. Stouffer, Dr. E. H. Taylor, Professor W. D. A. Westfall, Professor K. P. Williams.

At the dinner at the American Annex Hotel on Tuesday evening forty-eight persons were present.

At the business meeting of the Chicago Section and the Southwestern Section held at the end of the morning session of Wednesday, the following action was taken:

It was decided to hold the next meeting of the Southwestern Section at the University of Nebraska on November 27. The 
following programme committee was elected: W. C. Brenke, chairman, H. L. Rietz, O. D. Kellogg, secretary.

The Chicago Section elected for the ensuing biennium, Professor R. D. Carmichael, chairman; Professor Arnold Dresden, secretary; Professor C. N. Moore, third member of programme committee.

A resolution thanking Professor E. J. Moulton for his services as acting secretary of the Chicago Section during 191819 was unanimously passed.

A resolution on the death of Professor Laenas Gifford Weld on November 28, 1919, was adopted by a rising vote.

The Chicago Section and the Southwestern Section passed a resolution expressing interest in an investigation of the possibility of cooperative work in pure mathematics and in the domain between mathematics and the related sciences, as suggested by the National Research Council.

At the joint meeting on Tuesday afternoon, at which Professor O. D. Kellogg, vice-president of Section A, presided, the following papers were read:

I. Professor G. D. BIRKhoff, retiring vice-president of Section A of the American Association for the Advancement of Science, "Recent progress in dynamics."

II. Professor G. A. BuIss, retiring chairman of the Chicago Section of the American Mathematical Society, "Some recent developments in the calculus of variations."

III. Mr. H. H. Platt, "A suggestion for the utilization of atmospheric molecular energy."

The sessions of Tuesday forenoon, Wednesday forenoon and afternoon were presided over by Professors Bliss, Birkhoff and Roever respectively. At these sessions the following papers were read:

(1) Professor C. E. Love: "Note on a class of singular integral equations."

(2) Dr. R. F. Borden: "On the adjoint of a certain mixed equation."

(3) Professor G. O. James: "On a philosophical aspect of the relativity theory of gravitation."

(4) Professor O. Schmiedel: "On the early history of the number concept."

(5) Mr. B. Z. LiNFIELD: "On the relation of the roots of a polynomial to the roots of its derivative." 
(6) Professor E. L. DoDD: "A comparison of the arithmetic mean with other weighted means under certain laws of error."

(7) Professor S. LEFsChetz: "Real abelian varieties."

(8) Professor N. Altshiller-Court: "On a pencil of nodal cubics."

(9) Professor N. Altshiller-Court: "On an orthocentric quadrilateral."

(10) Professor G. A. Millen: "Properties of the subgroups of an abelian prime power group which are conjugate under the group of isomorphisms."

(11) Professor G. A. Miller: "Groups generated by two operators of order three whose product is of order four."

(12) Professor H. L. RIETz: "Urn schemata as a basis for the development of correlation theory."

(13) Professor K. P. Williams: "Concerning a problem in the flow of heat."

(14) Professor Tomlinson Fort: "Note on Dirichlet and factorial series."

(15) Professor H. J. EtTlinger: "Boundary value problems with regular singular points."

(16) Professor H. J. Ettuinger: "Extension of an existence theorem for a non-self-adjoint boundary value problem."

(17) Professor G. C. Evans: "Divergent Fourier series and integral equations."

(18) Mr. L. L. Stemmley: "On the general class of series of the form $Y(x)=c_{0}+\Sigma c_{n} g(n x)$."

(19) Professor A. C. LuNN: "A principle of duality in thermodynamics."

(20) Professor R. L. Borger: "On the Cauchy-Goursat theorem."

(21) Professor A. B. CoBle: "Some irrational and transcendental aspects of the invariants of a ternary quartic."

(22) Professor A. B. CoBle: "The Cayley symmetroid and the abelian modular functions of genus four."

(23) Professor E. J. Wilczynski: "One-parameter families and nets of ruled surfaces and a new theory of congruences."

(24) Professor A. EMcH: "Intersections of straight lines and systems of plane algebraic curves and the surfaces generated by them."

(25) Professor Pierre Boutroux: "A family of multiform functions."

(26) Dr. G. F. McEwen: "Fluid temperature distributions 
deduced from sources, boundary conditions, fluid velocity and convection."

(27) Dr. G. F. McEwen: "Direct application of the idea of "random sampling" in estimating the precision of averages, and the significance of differences."

Dr. Borden was introduced by Professor Carmichael. The papers of Professor Love, Dr. Borden, Professor Schmiedel, Mr. Linfield, Professor Evans, Mr. Steimley, Professor Borger, Professor Wilczynski, Professor Emch, and Professor Boutroux were read by title. Abstracts of the papers follow below.

1. Professor Love's paper develops the elementary theory of the equation

$$
\varphi(x)=\frac{f(x)}{x^{a}}+\lambda \int_{a}^{\infty} \frac{K(x, t) \varphi(t)}{x^{a} t^{\beta}} d t,
$$

where $\varphi(x)$ is the unknown function, under the assumption that the functions $f(x)$ and $K(x, t)$ are bounded for $x \geqq a$, $t \geqq a$. This case possesses special interest on account of the fact that it can be solved by a direct extension of the method of Fredholm.

2. Dr. Borden considers with the equation

$$
\Delta f^{\prime}(x)+a(x) f^{\prime}(x)+c(x) f(x)=0
$$

an adjoint equation. The invariants of the two equations under the group $f(x)=v(x) g(x)$ are compared, and also the invariants of the successive transforms of the two equations by the Laplace-Poisson transformations. (Poisson, Journal de l'Ecole Polytechnique, volume 6 (1806), pages 127-141.) Conditions are obtained for solutions of both equations in finite form and some remarks are made concerning the self-adjoint case.

3. The success of Einstein's theory of gravitation in removing the outstanding difference between theory and observation in the secular motion of the perihelion of Mercury, and its applicability to other problems of dynamical astronomy are used by Professor James as an argument in support of Bergson's thesis that there is no intrinsic order in nature and that the apparent mathematical order of the external universe has been imposed thereon by the intellect. 
4. In this paper Professor Schmiedel contends that the first appearance of the idea of extension of the number concept is found in the Ahmes papyrus.

5. Maxime Bôcher (Annals of Mathematics, 1892) gave a geometrical proof of the theorem of Lucas (Journal de l'Ecole Polytechnique, 1879), that the roots of the derivative of a cubic are the foci of the maximum ellipse inscribed in the triangle, whose vertices are the roots of the cubic, and asked the question "Could not the roots of the derivative of the general polynomial be brought into connection with the foci of the higher plane curves?" Mr. Linfield answers the question by proving that the roots of the derivative of a polynomial of degree $n$ are the foci of a curve of class $n-1$ which touches the segments joining the roots of the polynomial, two and two, at their midpoints. He generalizes and proves that the roots of the derivative $f^{\prime}(z)$ of the function $f(z)=\Pi$ $\left(z-z_{r}\right)^{\mu_{r}}$ are the foci of a curve of class $n-1$ which touches the segment $z_{i} z_{j}$ at a point dividing it in the ratio $\mu_{i}: \mu_{j}$.

6. Professor Dodd finds that if the probability that the error of a measurement will lie between $x$ and $x+d x$ is $c\left(1+|h x|^{3 / 2}\right)^{-1} d x$, with $c$ and $h$ constant, then the arithmetic mean of two measurements is the least probable value among all possible weighted means. Under the law $c_{1}\left(1+h^{2} x^{2}\right)^{-1}$, however, the probability that any weighted mean, including the arithmetic mean, will differ from the true value by less than $\epsilon$, is a constant, alike independent of the system of weights and the number of measurements. But under the laws $c_{2}\left[\left(1+h^{2} x^{2}\right)\left(1+k^{2} x^{2}\right)\right]^{-1}, h$ and $k$ equal or unequal, and $c_{3}$ $\left(e^{h x}+e^{-h x}\right)^{-1}$, the arithmetic mean is more reliable than any other weighted mean. The curves representing the foregoing laws of error closely resemble curves of the family $y=c_{4} e^{-h^{2} x^{2}}$, the probability function. The possibility of the arithmetic mean being a least probable value is associated with the fact that under certain laws of error the arithmetic mean of measurements is not as reliable as a single measurement. With an increase of the number of measurements, there is an increase in the probability that the larger errors will appear; and this may more than offset the neutralizing effect of errors of opposite sign. 
7. In this note, Professor Lefschetz shows how the number of algebraically distinct real hypersurfaces belonging to real abelian varieties may be obtained from a certain period matrix which characterizes them. This is followed by the actual determination of the number in question for a large class of varieties defined by the possession of certain complex multiplications.

8. Professor Altshiller-Court's first paper appeared in full in the February BuLletin.

9. The three vertices of a triangle and its orthocenter are the four vertices of an orthocentric quadrilateral having the property, pointed out by Carnot, that each vertex is the orthocenter of the triangle determined by the other three vertices. The four triangles thus formed, often referred to as an orthocentric group of triangles, have the same orthic triangle, and consequently the same nine-point circle, which may therefore be considered as belonging to the quadrilateral itself. Professor Altshiller-Court shows that the four circumcenters of an orthocentric group of triangles form another orthocentric quadrilateral, the two quadrilaterals having the same ninepoint circle, the center of which is a center of symmetry of the two orthocentric groups. The four centroids of the given orthocentric group of triangles also form an orthocentric group, the two groups having the same nine-point center, which point is a center of similitude of the two orthocentric groups, the ratio of similitude being -3 .

10. It is well known that if an abelian group $G$ has a subgroup of a given type, it has also a quotient group of this type and vice versa. If $G$ is the abelian group of order $p^{m}$ and of type $\left(m_{1}, m_{2}, \ldots m_{\lambda}\right)$ two of its subgroups $H_{1}$ and $H_{2}$ may be said to be of complementary types if each of them is simply isomorphic with the quotient group of $G$ with respect to the other. A necessary and sufficient condition that two subgroups of types $\left(m_{1}{ }^{\prime}, m_{2}{ }^{\prime}, \ldots, m_{\lambda}{ }^{\prime}\right)$ and $\left(m_{1}{ }^{\prime \prime}, m_{2}{ }^{\prime \prime}, \ldots\right.$, $\left.m_{\lambda}{ }^{\prime \prime}\right)$ are complementary is that each of the following equations is satisfied: $m_{1}^{\prime}+m_{1}^{\prime \prime}=m_{1}, m_{2}^{\prime}+m_{2}^{\prime \prime}=m_{2}, \ldots, m_{\lambda}^{\prime}+$ $m_{\lambda}{ }^{\prime \prime}=m_{\lambda}$. The number of the different types of subgroups which are complementary to the subgroups of a given type is equal to the number of the different sets of $I$-conjugate sub- 
groups of $G$ which are simply isomorphic with the subgroups of the given type, $I$-conjugate subgroups of $G$ being subgroups which are conjugate under its group of isomorphisms. The main object of Professor Miller's paper is to establish some fundamental reciprocal properties of the sets of $I$-conjugate subgroups contained in certain types of abelian groups of order $p^{m}$. Among the theorems established is the following: If all the invariants of an abelian group $G$ of order $p^{m}$ are equal to each other, then the number of its subgroups of a given type is equal to the number of its subgroups which are simply isomorphic with the quotient group of $G$ with respect to one of the former subgroups. In particular, the number of the distinct subgroups of order $p^{\alpha}$ contained in $G$ is equal to the number of its distinct subgroups of index $p^{a}$.

11. It is known that two operators $s_{1}, s_{2}$ of order 3 whose product is of order 4 generate a group of infinite order unless these operators are otherwise restricted. In the present paper, Professor Miller considers the groups generated by $s_{1}, s_{2}$ when the conditions imposed on these operators are as follows: $s_{1}{ }^{3}=s_{2}{ }^{3}=\left(s_{1} s_{2}\right)^{4}=\left(s_{1}{ }^{2} s_{2}\right)^{k}=1, k=3,4,5$. When $k=3$, these operators generate the group of order 48 obtained by extending the direct product of two cyclic groups of order 4 by means of an operator of order 3 which transforms this direct product into itself but is not commutative with any of its operators besides identity. When $k=4$, the group generated by these operators is the simple group of order 168 and a new and useful definition of this important group is thus obtained. Finally, when $k=5$, these operators generate a group of order 1,080 which is isomorphic with the simple group of order 360 with respect to its central. This group of order 1,080 does not contain the simple group of order 360 as a subgroup, but to each operator of the central quotient group there corresponds at least one operator of the group whose order is equal to the order of the corresponding operator in this quotient group.

12. In the present paper, Professor Rietz generalizes certain results of his first paper on this subject. In particular, the urn schema of the first paper that gives $t / s$ for the correlation coefficient, where $s$ is the number of balls in each of a pair of drawings, and $t$ the number of balls taken at random 
from the first drawing of a pair to be common to the pair, involves the hypothesis that the urn should be so maintained that the probability of drawing a white ball is $1 / 2$. This restriction is removed in the present paper so that the probability of drawing a white ball may be any constant $p$. The results are as simple as when $p=1 / 2$, and the proofs are but slightly more complicated.

13. A classic problem in the mathematical theory of the flow of heat is to determine the temperature of a semi-infinite solid, initially at the temperature zero, whose plane face is kept at a temperature that varies in a known manner with the time. The solution usually given is that obtained by Duhamel and followed by Riemann. It treats the problem as a limiting case of a simpler one. In the method used by Professor Williams, a definite integral is assumed for a solution. It is suggested by the solution of a related problem on noticing an interchange of boundary conditions. The integrand and limits contain arbitrary elements. These are determined by substituting the assumed solution in the differential equation that it must satisfy.

14. In this note Professor Fort exhibits a series which includes both Dirichlet and factorial series as special cases. He proves a fundamental convergence theorem.

15. The recent study of boundary value problems has been confined to the case where the coefficients of the differential equation are continuous throughout the interval of definition. Except for Porter's and Bôcher's work on the solutions of a differential equation having regular singular points (Bôcher, "Oscillation theorems of Sturm," this Bulletin, volume 5 (1898)), no attention has been given to the general theory of existence of solutions, oscillation theorems and expansions in normal functions for a system such as occurs in applied problems involving Legendre's, Bessel's or Lamé's differential equations. In this paper, Professor Ettlinger shows that a number of the theorems which hold for the continuous system go over for the system with regular singular points.

16. In a paper to appear in the June number of the Annals of Mathematics, Professor Ettlinger establishes the existence of 
at least one real characteristic number for the system

$$
\begin{gathered}
\frac{d}{d x}\left[K(x, \lambda) \frac{d u}{d x}\right]-G(x, \lambda) u=0, \\
A_{i 1} u(a)-A_{i 2} K(a) u_{x}(a)-A_{i 3} u(b)+A_{i 4} K(b) u_{x}(b)=0, \\
(i=1,2),
\end{gathered}
$$

where the $A_{i j}$ are constants and certain conditions are satisfied by $K$ and $G$. This theorem is now extended to a system of the same form where $A_{i j}(\lambda)$ replaces the constant coefficients of the boundary conditions, subject to the additional restriction that $\left(A_{1 i} A_{2 j}-A_{2 i} A_{1 j}\right) /\left(A_{1 k} A_{2 l}-A_{2 k} A_{1 l}\right)$ decrease (or do not increase) as $\lambda$ increases, for specified values of $i, j, k, l$.

17. Professor Evans's paper is an endeavor to connect by practical calculation the concepts of divergent series, integral equation, and Stieltjes integral. The equation treated is equation (1) in Lecture $\mathrm{V}$ of the author's Colloquium Lectures, when the kernel is of the form $f(x+s)+g(x-s)$, with $f$ and $g$ periodic with period $a b$ as functions of a single argument. The solution is developed in Fourier series. An "imaginary" resolvent kernel is obtained in the form of a Fourier series, generally divergent and not even summable, which nevertheless yields with proper choice of the known function a summable series as solution of the equation, and thus affords a practical method of handling such equations. The equation treated, which is of the first kind, includes the equation of the second kind as a particular case.

18. In this paper, Mr. Steimley considers the general class of series of the form

$$
Y(x)=c_{0}+\sum_{n=1}^{\infty} c_{n} g(n x),
$$

where $g(x)$ has the asymptotic character

$$
g(x) \sim x^{P(x)} e^{Q(x)}\left(1+\frac{a_{1}}{x}+\frac{a_{2}}{x^{2}}+\cdots\right),
$$

which is valid for $x$ approaching infinity in some sector $V$ bounded by two rays extending from zero to infinity. $P(x)$ 
and $Q(x)$ are polynomials in $x, a_{i}(i=1,2, \cdots)$ are functions of the angle $\theta$, which is the argument of $x$, varying in the sector $V, c_{i}(i=0,1,2, \cdots)$ are independent of $x$. The character of the regions of convergence, of absolute convergence and of uniform convergence are determined. The character of the points on the boundary of the region of convergence is determined, except for an isolated set of points. The character of these isolated points is determined except in very special cases.

A necessary and sufficient condition that $Y(x)$ shall converge is determined. The uniqueness of expansion of functions in terms of $Y(x)$ is determined under special conditions.

19. In this paper, Professor Lunn shows that the general relations in thermodynamics can be arranged and the notations adjusted so as to show a complete duality analogous to the similar principles already known in geometry, mechanics and electromagnetism. Energy is dual to entropy and temperature to its own reciprocal, and a corresponding ranging in pairs occurs throughout the range of quantities characteristic of the thermodynamic properties of material systems.

As illustrations of the working of the principle various applications are suggested to the dual representation of physical relations and the dual interpretations of mathematical relations, to the attainment of symmetry of notation, to the transformation of the thermodynamic potentials, and to the criteria of equilibrium and stability.

20. In this paper Professor Borger proves, by using the Denjoy integral, that if the two functions $U(x, y), V(x, y)$ of the real variables $x, y$ are continuous in these variables jointly and possess finite partial derivatives of the first order, satisfying the Cauchy-Riemann differential equations at each point of the simply connected closed region $R$, then $U$ and $V$ are analytic functions of these variables.

An application to the theory of functions of a complex variable shows that if $W=U(x, y)+i V(x, y)$ and if $U$ and $V$ satisfy the conditions above referred to, then $W^{\prime}$ exists and is analytic.

21. The generalized Kummer surface is, for the genus three, a 3-way, $M_{3}$, in $S_{7}$ obtained by equating the coordinates to 
linearly independent theta functions of the second order and zero characteristic in three variables. Professor Coble shows that $M_{3}$ is the double manifold on a quartic locus $Q_{6}$ in $S_{7}$. The fifteen coefficients of $Q_{6}$, which are modular functions, satisfy a system of 63 cubic relations. On the other hand it is well known that seven points in a plane define a ternary quartic with isolated double tangents. Professor Coble has shown that the simplest linear system of invariants of degree three in the coordinates of each of the seven points has fifteen linearly independent members. These also satisfy a system of 63 cubic relations and are shown to be identical with the coefficients of $Q_{6}$ above. The modular group induces on these coefficients a group $G$ of linear transformations. The invariants of $G$ are invariants of the ternary quartic, which can be expressed either irrationally in terms of the seven points or transcendentally in terms of the theta moduli.

22. By combining some theorems of Schottky with some results of his own, Professor Coble proves the following theorems concerning the Cayley symmetroid (a certain tennodal quartic surface with nine absolute constants): (1) The coordinates of the ten nodes of the symmetroid can be expressed uniformly by means of abelian modular functions of genus four. (2) Under integer linear transformation of the periods the symmetroid is algebraically transformed by regular Cremona transformation. (3) In particular those period transformations which are congruent to identity modulo 2 leave the symmetroid unaltered.

It thus appears that the ten nodes of the symmetroid are related to the abelian modular functions of genus four as the eight base points of a net of quadrics to the functions of genus three.

23. In this paper, Professor Wilczynski thinks of a congruence of lines as the totality of the generators of a oneparameter family of ruled surfaces, basing the analytic theory upon the previously developed theory of ruled surfaces, and connecting this theory with the theory of congruences by a chain of several related theories which seem to be entirely new. The principal advantage gained for the theory of congruences consists in the new expressions found for the invariants, expressions which are elegant in form and directly 
applicable to a congruence whose developables are not known. Moreover the auxiliary quantities in terms of which these invariants are expressed are interpretable in terms of the new theories of one-parameter families of ruled surfaces, and nets of ruled surfaces.

24. According to Lüroth (Crelle's Journal, volume 68, pages 185-190) the analytic method of investigating problems of the sort here considered, as outlined by him and suggested by a paper of Clebsch, is too difficult on account of the complicated relations between the coefficients of the given and required elements and forms. Making use of a purely geometric method, Lüroth solves a number of problems in which the curves are conics.

Professor Emch shows that these and more general problems of this type may be solved by a relatively simple analytical method which consists chiefly in the utilization of a singular null system of the following type:

$$
\begin{aligned}
& \rho x_{1}=0+\alpha_{2} p_{12}+\alpha_{3} p_{13}+\alpha_{4} p_{14}, \\
& \rho x_{2}=-\alpha_{1} p_{12}+0+\alpha_{3} p_{23}+\alpha_{4} p_{24}, \\
& \rho x_{3}=-\alpha_{1} p_{13}-\alpha_{2} p_{23}+0+\alpha_{4} p_{34}, \\
& \rho x_{4}=-\alpha_{1} p_{14}-\alpha_{2} p_{24}-\alpha_{3} p_{34}+0 .
\end{aligned}
$$

For $i=1,2,3,4 ; k=1,2,3,4 ; i \neq k$, the $x_{i}$ are the coordinates of a point, the $\alpha_{i}$ those of a plane, the $p_{i k}$ those of a straight line. The determinant of the transformation (null system $=$ involutory correlation throughout) is

$$
\left(p_{12} p_{34}+p_{13} p_{42}+p_{14} p_{23}\right)^{2}=0 \text {. }
$$

This null system is therefore singular and has the following geometric meaning. The plane $\left(\alpha_{i}\right)$ cuts the straight line $\left(p_{i k}\right)$ in a point $\left(x_{i}\right)$ whose coordinates are precisely those given by (1). The method based upon this system is believed to be new.

25. Professor Boutroux considers the following fundamental problem in the study of multiform functions satisfying differential equations of the first order. Calling $f(x, C)$ the general solution of an equation, find the values $C_{1}, C_{2}, \cdots$ of $C$ which belong to the same function (that is, are such that $f\left(x, C_{1}\right)$, 
etc., are branches of one and the same multiform function). The substitutions exchanging these values $C_{1}, C_{2}, \cdots$, form a discontinuous group $G$, and to investigate that group, we have to consider certain functions $\psi(C)$ (substituting functions). As a rule these last functions are themselves multiform functions. But some families of differential equations have the property that to build up the corresponding group $G$, it is not necessary to use the whole functions $\psi(C)$ but merely special branches or uniform "elements" of these functions which can be defined simply. Thus the difficulties arising from the multiform character of the functions in the problem are overcome.

As an example, one may take the functions $z(x)$ satisfying $z(d z / d x)=m z+2\left(x^{3}-1\right)$. Choosing for $C$ a parameter which determines $z$ at $x=\infty$, one first sees that all the substitutions of $G$ are of the form $\left[C, C+m \int_{\Gamma} z d x\right]$, where $\Gamma$ is any closed path starting from $x=\infty$. Now it is possible to find two special substituting functions of this type, $\psi_{1}, \psi_{2}$, each of which is holomorphic in the whole $C$-plane limited by two straight cuts (straight lines joining two certain points to $x=\infty$ ). Considering the said functions exclusively in these limited fields, one can define uniformly for all values of $C$, two substitutions $\left[C, \psi_{1}(C)\right]$ and $\left[C, \psi_{2}(C)\right]$. These particular substitutions, combined with a third one of the form $[C, C$ + constant] will be sufficient, if properly chosen, to build up the total group $G$.

26. The coefficient of heat conductivity of most fluids, determined from observations made under controlled conditions, in which motion is eliminated, is very small. Moreover, in order to apply the results of the classical theory of the flow of heat to fluids, they must be regarded as solids. In this paper, Dr. McEwen presents the derivation of differential equations of the flow of heat in a fluid due to a convective, or alternating circulation of small fluid volumes, both when the average fluid velocity across a given surface is zero, and when it has an appreciable value. Two sets of equations are derived; one for the relatively cold elements, the other for the warm elements. The temperature that would be indicated by a thermometer depends upon both the warm and cold elements since they are intermixed. The measurable tempera- 
ture is therefore a mean of these two, and a proper combination of the first two sets of equations results in a single set for determining this temperature.

27. The usual method of deducing frequency curves from assumptions as to the manner in which elementary errors or deviations arise often requires a larger number of measurements than are available in practical applications. Moreover, the difficulty of determining and handling the proper frequency curve except in special cases in which the Gaussian, or normal law holds, restricts the use of such deductive methods, and often results in erroneous applications of the simple normal law. By arranging the observations in their order of magnitude, and regarding two groups, those above the average and those below, as samples of corresponding groups of an indefinitely large sample, Dr. McEwen reduces the problem of the precision of averages to the simple one of variation in proportions. Regarding the observed average of the whole series as the best estimate of that of an indefinitely large sample, and changing the proportion of observations above and below the average, the probability of these variations in proportions corresponds to that of the resulting variation in the average computed from the new group values and the observed constant proportion. A frequency distribution of averages thus computed gives directly the probability that the true average falls within given limits. From similar determinations of the frequency distribution of the averages of two sets of observations, the probability can be found that the true values differ by an amount exceeding any specified value. A modification of this "group proportion" method not requiring the determination of the frequency distributions serves to estimate rapidly the probability that an observed difference between two averages arose by chance. The above results apply to any number of observations, and to any type of frequency distribution of the observations.

O. D. KeLloGg, Secretary of the Southwestern Section. ARNold DreSDEN, Secretary of the Chicago Section. 